\title{
Facile Synthesis of Nitrogen-doped Carbon Quantum Dots for Bio-imaging
}

\author{
Persia Ada N. de Yro ${ }^{1,2,3, a}$, Beejay T. Salon ${ }^{2}$, Blessie A. Basilia ${ }^{1,2}$, Mark Daniel de Luna ${ }^{3}$ and Peerasak Paoprasert ${ }^{4}$ \\ ${ }^{1}$ Materials Science Division, Industrial Technology Development Institute, Department of Science and Technology, DOST Compound, \\ Bicutan, Taguig City, Philippines \\ ${ }^{2}$ School of Graduate Studies, Mapua Institute of Technology, Muralla Street, Intramuros, Manila, Philippines \\ ${ }^{3}$ College of Engineering, University of the Philippines, Diliman, Quezon City, Philippines \\ ${ }^{4}$ Department of Chemistry, Faculty of Science and Technology, Thammasat University, Pathumthani, 12121 Thailand
}

\begin{abstract}
Carbon quantum dots (CQD) with fascinating properties has gradually become a rising star as a new nanocarbon member due to its nonthreatening, abundant and inexpensive nature. This study reports on a facile preparation of fluorescent carbon quantum dots (CQD) from iota Carrageenan. CQD from iota Carrageenan was produced by hydrothermal method with a quantum yield (QY) of 16 to $20 \%$. Doping the CQD with nitrogen by the addition of tetraethylene pentamine (TEPE) produced CQD with a QY of 77\%. FTIR data confirmed the formation of hydroxyl, carboxylic and carbonyl functional groups as confirmed by the ToFSIMS data due to the presence of nitrogen bonds on the N-CQD produced with TEPE. The CQD and N-CQD produced are crystalline with graphitic structures because of the presence of $\mathrm{sp} 2$ graphitic $d$ line spacing with the sizes ranging from 2 to $10 \mathrm{~nm}$. To examine the feasibility of using the CQD as nanoprobe in practical applications, labelling and detection of E.coli was performed. The E.coli fluoresced proving CQD as an effective probe in bio imaging application. This study has successfully demonstrated a facile approach of producing CQD with significant high quantum yields to fluorescent CQD for bio imaging applications.
\end{abstract}

\section{Introduction}

Carbon quantum dots (CQDs) are a new class of 'zerodimensional' nanomaterials in the carbon family with sizes below $10 \mathrm{~nm}$. They were first discovered during refinement of single-walled carbon nanotubes by preparative electrophoresis in 2004 and via laser ablation of graphitic powder and cement in 2006. CQDs exhibit non-blinking luminescence and superior water dispersability. More importantly, they have novel optical properties, outstanding biocompatibility and robust chemical inertness making it a potential material for optical nanoprobes in high contrast bio-imaging and biosensing applications, and their intense and tunable photoluminescence and high water solubility allows them to be used in catalysis and energy related fields. (Yi Liu, 2014;Liu, Qin, Zhang, Chao, Weihang, \& Chun, 2014 ;Hu, 2014;Mingbo Wu, 2014)

Carbon-based quantum dots (CQDs) have gradually become a rising star as a new nanocarbon member because of its fascinating properties comparable to semiconductor quantum dots (Baker \& Baker, 2010). These CQDs could be produced using carbon materials such as graphite, graphite oxide, nanodiamonds, carbon nanotubes, activated carbon, and carbon soot with different methods of synthesis such as laser ablation, electrochemical oxidation and arc discharge CQDs (Haitao, Zhenhui, Yang, \& Shuit-Tong, 2012).
Unfortunately these processes require costly precursors, complex instrumental set-ups or post-treatments.

There are numerous studies on the synthesis of CQDs from natural resources but, to date, there are none reported from the Philippines. With the abundance of natural resources in the Philippines, seaweed as a source of iota carrageenan in particular, it is practical that alternative uses be explored. In addition to this, CQDs have numerous potential applications thus the need to search for a cheaper source, and cheaper, safer and faster mode of synthesis. This paper reports about hydrothermal synthesis and characterization of CQD from iotacarrageenan. It will also report the effect of doping the CQD with nitrogen. The response variable to be observed is the quantum yield and the color of quantum dot produced. The produced CQD will be tested for its viability to be applied in bio-imaging.

\section{Experimental}

\subsection{Materials}

The study used an analytical grade iota-carrageenan purchased from Sigma-Aldrich (Singapore). The iotacarrageenan contains two sulphates per disaccharide. Sulfuric acid and Sodium Chloride were purchased from Carlo Erba reagents company (Val-de-Reuil, France).

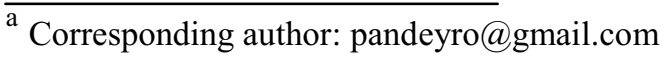


Tetraethylene pentamine (TEPE) were purchased from Merck ( Hohenbrunn, Germany). All solvent and reagent are all analytical grade.

\subsection{Apparatus}

Fourier transform infrared spectroscopy (FTIR) Perkin Elmer Frontier Simultaneous Thermal Analyzer (STA) 6000 was used to characterise the spectra from $600 \mathrm{~cm}-1$ to $4000 \mathrm{~cm}-1$ for the produced carbon quantum dots in determining the presence functional groups present hydroxyl, epoxy, carbonyl, and carboxylic acid groups. IonTOF Time of Flight Secondary Ion Mass Spectroscopy positive and negative spectra were used to detect the functional bonds and compounds formed in the CQD and N-CQD. Samples for ToFSIMS analysis were prepared by dispersing the particles in solution by sonication. The dispersion was placed drop wise on a silicon wafer. Transmission Electron Microscopy (TEM) analysis was performed using $200 \mathrm{kV}$ JEM-2100F FETEM with to determine the average particle size, and morphology and crystallinity of the produced CQD. Samples for TEM characterization were prepared by dispersing the particles in solution by sonication. The dispersion was placed drop wise on Holey Carbon coated copper grids. UV-Vis spectrophotometer Perkin Elmer Lambda 35 was used to determine the absorbance behaviour of CQDs under UV-Vis spectra. 500 micro litres of samples were placed in the quartz glass and the standard procedure was followed accordingly (Perkin Elmer). Deionised water was used to perform a correction (blank) in the spectrum of the CQDs. Microplate testing was performed using microplate Thermo Scientific Varionskan Flash (white plate). Samples were placed in the white microplate with excitation wavelength of $310 \mathrm{~nm}-500 \mathrm{~nm}$.

\subsection{Procedure}

\subsubsection{Synthesis of carbon quantum dots by hydrothermal method}

Carrageenan powder was dissolved in sulfuric acid and autoclaved. The solution was filtered and centrifuged at 12,000 RPM to remove. The CQD was freeze dried and stored for characterization and testing.

\subsubsection{Hydrothermal reaction using passivating compound Tetraethylene pentamine (TEPE)}

Carrageenan powder was dissolved in TEPE, deionized and autoclaved. The solution was filtered and centrifuged at 12,000 RPM to remove. The CQD was freeze dried and stored for characterization and testing.

\subsection{Bio-imaging}

The produced CQD were tested for bio-imaging application. E-coli bacteria were placed in a sterilised microscope slides following incubation for 3 hours at room temperature. Following the study by (Mehta, Jha and Kailasa) where standard procedures in staining and simple smears of e-coli bacteria with CQDs were performed. Bio imaging of e-coli samples with and without CQDs as a probe was characterized in Olympus BX51 Fluorescence Microscope.

\section{Results and discussion}

\subsection{Optimization of Synthesis of CQD}

In the production of CQD via hydrothermal process, we were able to produce three distinct colors (yellow, green and blue). At lower acidity of $1 \%$ concentration and lower temperatures of $180^{\circ} \mathrm{C}$ for 12 hours, yellow CQDs are produced with a $16.9 \%$ QY while at acid content of $1 \%$ and reaction time of $4 \mathrm{hrs}$ and temperature of $250^{\circ} \mathrm{C}$, green CQDs with the QY of $17.5 \%$. Statistical analysis, regression and parametric interaction for time and temperature show that the optimum time is 4 hours and optimum temperature is at $250^{\circ} \mathrm{C}$. The surface plot of the results of the quantum yield (Figure 1) verifies that at higher temperature with lower time similar to the results obtained by Bhunia et al, 2013, where increasing the rate of carbonization will promote rapid nucleation over growth rate.

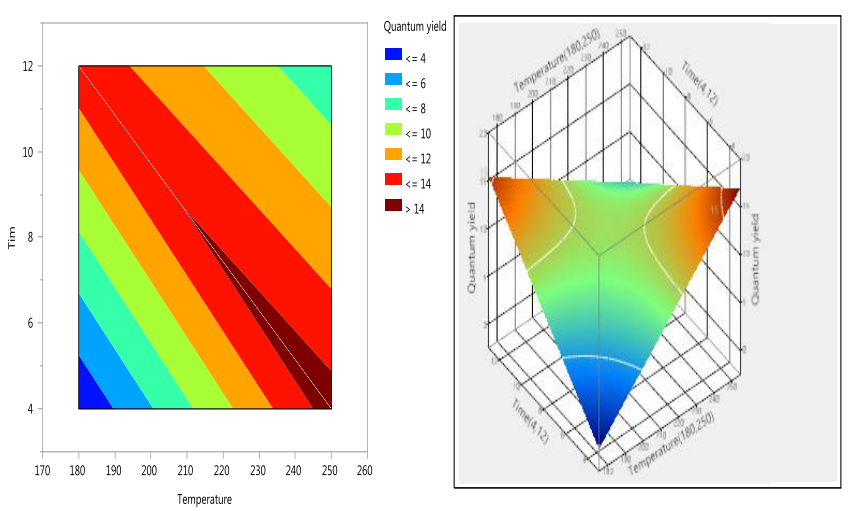

Figure 1. Contour and Surface profile of the effect of Temperature and Time on the Quantum Yield

\subsection{Characterization of CQD and N-CQD}

FTIR data signifies the formation of aromatic rich compounds with $\mathrm{C}=\mathrm{C}$ functional groups were observed in samples with surface passivation which could be attributed to the asymmetric and symmetric stretching vibration of $\mathrm{COO}$ - respectively. Peaks ranging from $1600 \mathrm{~cm}^{-1}$ to $1500 \mathrm{~cm}^{-1}$ can be considered as $\mathrm{C}=\mathrm{O}$; on the other hand, functional group of $\mathrm{O}-\mathrm{H}$ can be observed at peaks $3000 \mathrm{~cm}^{-1}$ to $3500 \mathrm{~cm}^{-1}$ As a result, the samples were composed of hydroxyl and carboxylic/carbonyl moieties which might have originated from the carbohydrates in the citrus peel making the sample rich in electron functional groups. 


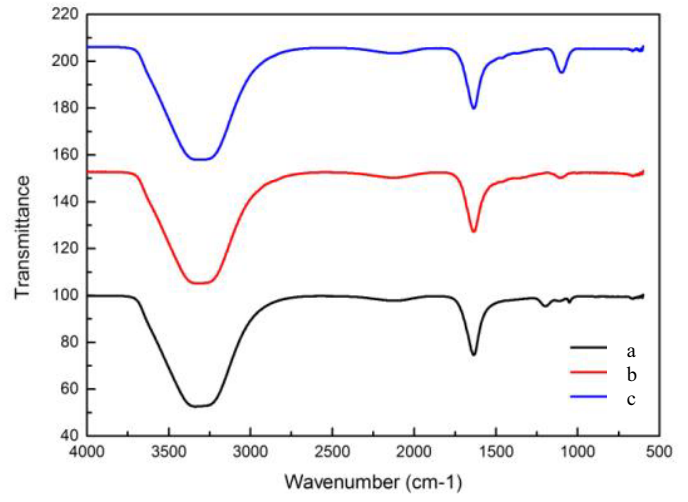

Figure 2. FTIR data for CQD produced under the following conditions (a) $1 \%$ acid heated at $180^{\circ} \mathrm{C}$ for 12 hours (b) $4 \mathrm{ml}$ TEPE heated at $250^{\circ} \mathrm{C}$ for 4 hours and (c) $1 \%$ acid with TEPE heated at $180^{\circ} \mathrm{C}$ for 12 hours.

ToFSIMS negative spectrum data indicates the formation of nitrogen bonds on the CQD produced with TEPE which explains the increase in quantum yield similar to the results obtained in previous studies. The CQD produced was crystalline with graphitic structures because of the presence of sp2 graphitic $d$ line spacing with the sizes ranging from 2 to $10 \mathrm{~nm}$.
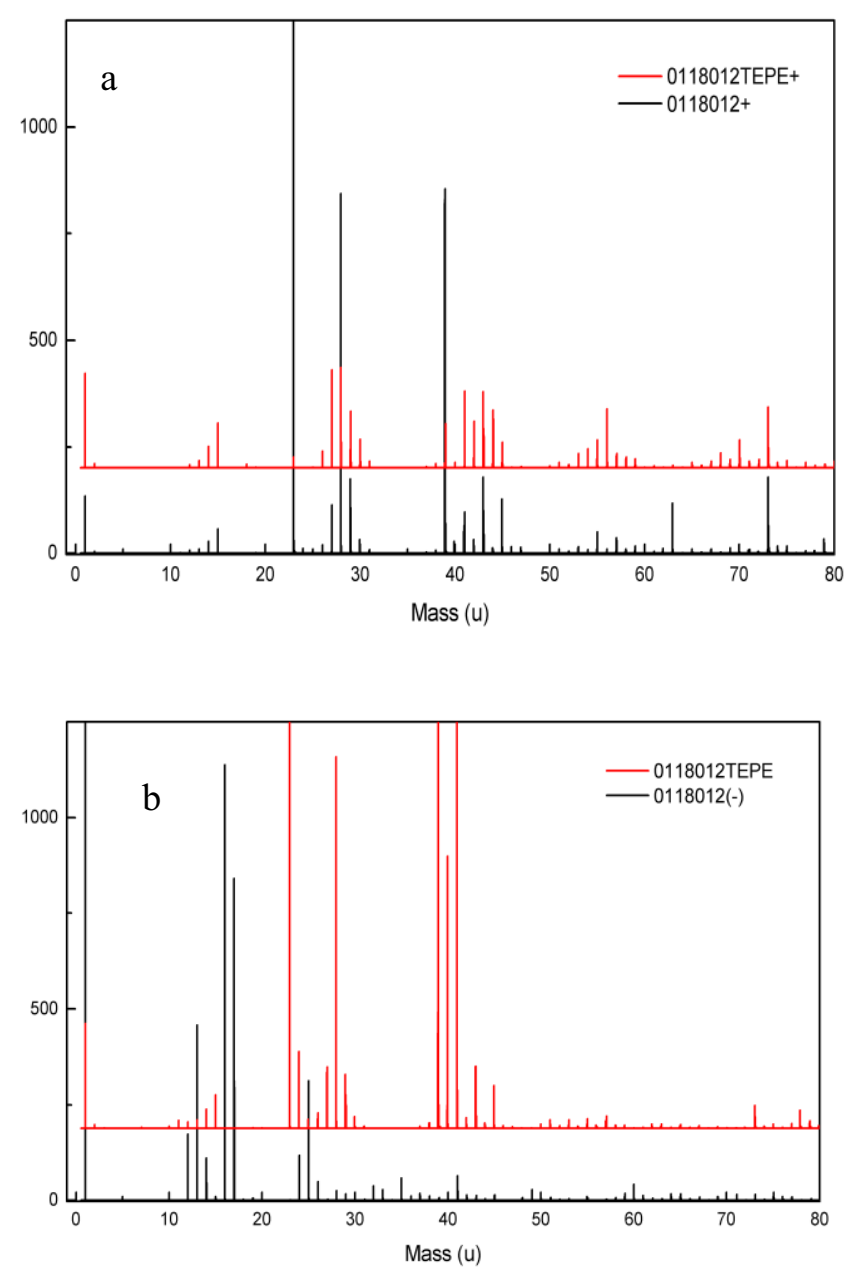

Figure 3. ToFSIMS (a) positive spectra and (b) negative spectra data for CQD produced under the following conditions (red) $1 \%$ acid heated at $180^{\circ} \mathrm{C}$ for 12 hours and (black) $1 \%$ acid with TEPE heated at $180^{\circ} \mathrm{C}$ for 12 hours

High resolution TEM images show that the CQD produced have crystalline structure with lattice spacing of $0.312 \mathrm{~nm}(3.12 \AA)$ which may be recognized to the (102) diffraction planes of graphitic ( $\mathrm{sp} 2)$ carbon

\subsection{Photoluminescence properties of CQD and N-CQD}

The PL data in Figure 4 show that CQD with different sizes emit specific wavelengths under UV lamp. Figure 4(a) is the data from the sample prepared with $1 \%$ acid heated at $180^{\circ} \mathrm{C}$ for 12 hours, it can be seen that the particle size ranges from 6-10 $\mathrm{nm}$ which corresponds to a wavelength of 425 to $600 \mathrm{~nm}$ emitting a yellow fluorescence; Figure 4(b) was synthesized with $1 \%$ acid and TEPE heated at $180^{\circ} \mathrm{C}$ for 12 hours have a particle size distribution of $4-7 \mathrm{~nm}$ with corresponding wavelength from $400-600 \mathrm{~nm}$ and emitting green fluorescence; Figure 4(c) which was synthesized with $4 \mathrm{ml}$ TEPE heated at $250^{\circ} \mathrm{C}$ for 4 hours has a particle size distribution of 2$6 \mathrm{~nm}$ with corresponding wavelength from $400-50 \mathrm{~nm}$ and emitting blue fluorescence. Doping the CQD with nitrogen by the addition of tetraethylene pentamine (TEPE) produced CQD with a QY of $77 \%$ as compared to those produced without TEPE which produced a QY of $16.9 \%$. This confirms that doping with $\mathrm{N}$ increases the QY which agrees with the results of Hui Ding, et al, 2014 and Xiaojuan Gong, 2015 where they concluded that N-S co-doping has the highest QY owing to the synergistic effect of nitrogen-sulfur co- doping.
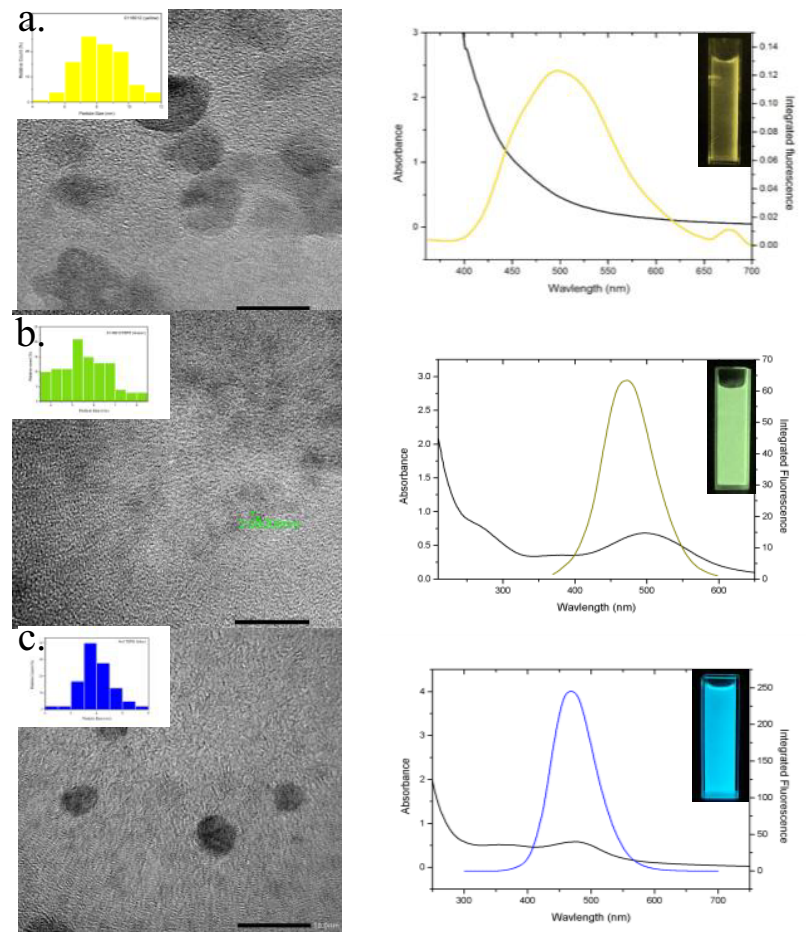
Figure 4. TEM, particle size distribution analysis by Image $\mathrm{J}$ and PL data of CQD produced under the following conditions (a) $1 \%$ acid heated at $180^{\circ} \mathrm{C}$ for 12 hours (b) $1 \%$ acid with TEPE heated at $180^{\circ} \mathrm{C}$ for 12 hours and (c) $4 \mathrm{ml} \mathrm{TEPE} \mathrm{heated} \mathrm{at}$ $250^{\circ} \mathrm{C}$ for 4 hours.

\subsection{Bio-imaging of CQD and N-CQD}

Carbon quantum dots were used as a substitute for staining the bacteria base on the study performed by Mehta, Jha and Kailasa. Figure 5 shows the large number of functional groups, mainly carboxyl groups in the surface of the CQDs, contributed to the positive and negative attraction on the bacteria when ionized. With this positive and negative charges bond, the bacteria was stained creating an ionic bond. Staining microbial cells is important because without stain, most bacterial cells are extremely difficult to see. Staining allows them to be seen so that observations as to their morphology (i.e., individual cell shape) and arrangement (i.e., how the cells remain physically attached to one another after cell division) can be made. The chemical substance commonly used to stain bacteria is called dye. The dyes are in solutions called stains and can be acidic in terms of nature.
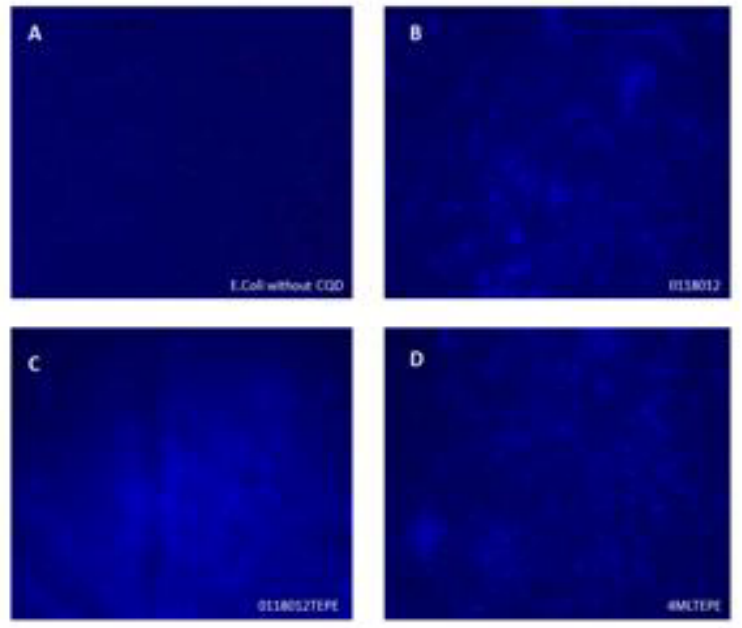

Figure 5. E.coli detection fluorescence image (a.)E.coli without CQDs, produced under the following conditions (b) $1 \%$ acid heated at $180^{\circ} \mathrm{C}$ for 12 hours (c) $1 \%$ acid with TEPE heated at $180^{\circ} \mathrm{C}$ for 12 hours and (d) $4 \mathrm{ml}$ TEPE heated at $250^{\circ} \mathrm{C}$ for 4 hours.

\section{Conclusion}

In summary, this study has successfully demonstrated a facile method of producing CQD from iota carrageenan. CQD quantum yield from 16 to $17 \%$ were obtained from the response optimization parameter for synthesis of CQD by hydrothermal method. It was also proven that doping the CQD with nitrogen by the addition of tetraethylene pentamine (TEPE) produced N-CQD with a QY of $77 \%$. This is in agreement with the results of previous studies where they claim that the doping of $\mathrm{N}$ in CQDs facilitate the growth of carbon core, allowing the formation of larger N-CQDs (Hui Ding, et al, 2014 and Xiaojuan Gong, 2015). FTIR data confirmed the formation of hydroxyl, carboxylic and carbonyl functional groups. The formation of N-CQD was confirmed by the ToFSIMS data due to the presence of nitrogen bonds on the N-CQD produced with TEPE. The CQD and N-CQD produced are crystalline with graphitic structures because of the presence of $\mathrm{sp}^{2}$ graphitic $\mathrm{d}$ line spacing with the sizes ranging from 2 to $10 \mathrm{~nm}$. To examine the feasibility of using the CQD as nanoprobe in practical applications, labelling and detection of E.coli was performed. The E.coli fluoresced proving CQD as an effective probe in bio imaging application. This study has successfully demonstrated a facile approach of producing fluorescent CQD with significant high quantum yield for bio imaging applications.

\section{References}

1. Baker, S. N., \& Baker, G. N. (2010). Luminescenct Carbon Nanodots: Emergent Nanolights. Angew. Chem International Edition, 6726-6744.

2. Haitao, L., Zhenhui, K., Yang, L., \& Shuit-Tong, L. (2012). Carbon nanodots: synthesis, properties and applications. Journal of Materials Chemistry, DOI: $10.1039 / \mathrm{c} 2 \mathrm{jm} 34690 \mathrm{~g}$.

3. Hui, D., Ji-Shi W. and Huan-Ming X. (2014). Nitrogen and sulfure co-doped carbon dots with strng blue luminescence.Royal Society of Chemistry 2014.

4. Hu, Y. W. (2014). Carbon quantum dots: synthesis, properties and applications. J. Mater. Chem C, 69216939.

5. Liu, H., Q. W., Zhang, C., C. L., W. J., \& C. W. (2014). A multifunctional ribonuclease A-conjugated carbon dot cluster nanosystem for synchronous cancer imaging and therapy. Nano Research Letters, 9-3-97.

6. Mingbo Wu, Y. W. (2014). Preparation of functionalized water-soluble photoluminescent carbon quantum dots from petroleum coke. Carbon 78, 480-489.

7. Xiaojuan Gong, W. L. (2015). Facile Synthesis of nittrogen-doped carbon dots for $\mathrm{Fe}+3$ sensing and cellular imaging. Analytica Chimica Acta.

8. Yi Liu, N. X. (2014). One-step microwave-assisted polyol synthesis of green luminescent carbon dots as optical nanoprobes. Carbon 68, 258-264. 